\title{
Solvation of N,C-Protected Valine: Interactions with DMSO and a Chiral Solvating Agent
}

Karoline Bünnemann, Christian Merten*

Organic Chemistry 2, Ruhr-University Bochum, 44801 Bochum, Germany.

\section{Contents}

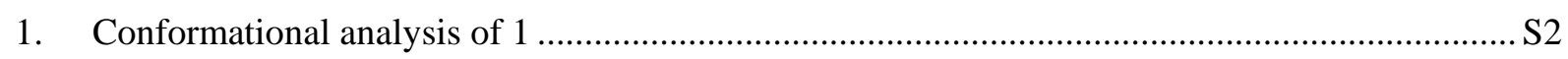

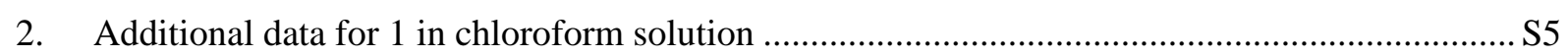

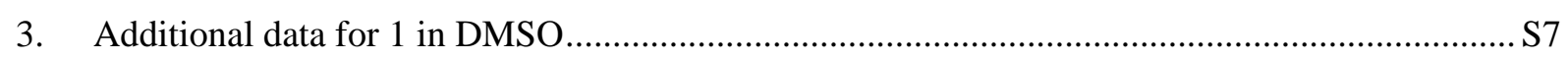

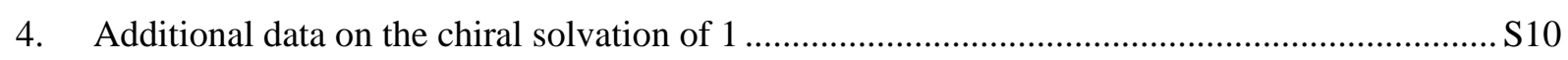

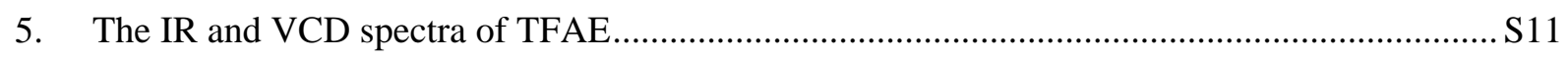

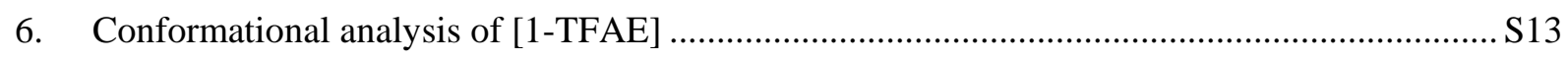




\section{Conformational analysis of 1}

Table S1. Summary of conformational analysis of $\mathbf{1}$ including relative zero-point corrected and Gibbs free energies, $\Delta \mathrm{E}_{\mathrm{ZPC}}$ and $\Delta \mathrm{G}_{298 \mathrm{~K}}$, the corresponding Boltzmann populations, and important torsional angles.

\begin{tabular}{|c|c|c|c|c|c|c|c|c|c|}
\hline Conf. & $\begin{array}{l}\Delta \mathbf{E}_{\mathrm{ZPC}} \\
\left(\mathrm{kcal} \mathrm{mol}^{-1}\right)\end{array}$ & $\begin{array}{l}\Delta \mathbf{G}_{298 \mathrm{~K}} \\
\left(\mathrm{kcal} \mathrm{mol}^{-1}\right)\end{array}$ & $\begin{array}{l}\text { pop- } \Delta E \\
(\%)\end{array}$ & $\begin{array}{l}\text { pop- } \Delta G \\
(\%)\end{array}$ & $\phi$ & $\psi$ & $\alpha$ & $\rho 1$ & $\rho 2$ \\
\hline Beta & 0.08 & 0.00 & 6.59 & 20.73 & -105.96 & 121.55 & 176.27 & -164.84 & -179.40 \\
\hline Beta-1-2 & 0.73 & 0.84 & 2.21 & 5.01 & -105.47 & 119.12 & 176.82 & -144.99 & 66.29 \\
\hline Beta-1-3 & 0.90 & 1.44 & 1.66 & 1.81 & -109.04 & 124.02 & 175.77 & -160.23 & -66.74 \\
\hline Beta-2 & 0.41 & 1.04 & 3.79 & 3.55 & -119.93 & 157.23 & -64.23 & -163.44 & -179.49 \\
\hline Beta-2-3 & 1.22 & 2.20 & 0.97 & 0.51 & -121.90 & 158.56 & -64.20 & -162.54 & -66.67 \\
\hline Beta-3 & 0.73 & 1.07 & 2.19 & 3.42 & -143.93 & 149.68 & 66.13 & -175.49 & -179.74 \\
\hline Beta-3-2 & 1.45 & 2.41 & 0.65 & 0.36 & -143.15 & 147.96 & 65.18 & -171.91 & 66.73 \\
\hline Beta-4 & 1.13 & 2.32 & 1.13 & 0.41 & -146.38 & 151.23 & 66.72 & 92.06 & 63.76 \\
\hline Beta-4-1 & 1.18 & 2.12 & 1.03 & 0.58 & -144.42 & 149.31 & 65.58 & 116.66 & -65.48 \\
\hline Beta-4-2 & 0.61 & 1.63 & 2.67 & 1.32 & -144.75 & 151.09 & 67.13 & 96.12 & 179.43 \\
\hline Beta-5 & 0.00 & 0.50 & 7.54 & 8.94 & -109.50 & 123.97 & 175.78 & 96.36 & 179.60 \\
\hline Beta-5-2 & 0.50 & 0.93 & 3.25 & 4.28 & -108.74 & 123.44 & 175.93 & 92.73 & 64.27 \\
\hline Beta-5-3 & 0.52 & 1.01 & 3.12 & 3.79 & -113.48 & 125.49 & 175.66 & 120.80 & -65.53 \\
\hline Beta-6 & 0.29 & 1.30 & 4.60 & 2.30 & -121.60 & 157.91 & -63.69 & 95.54 & 179.62 \\
\hline Beta-6-2 & 0.85 & 1.91 & 1.78 & 0.83 & -121.04 & 156.98 & -64.34 & 118.08 & -65.32 \\
\hline Beta-6-3 & 0.78 & 1.93 & 2.01 & 0.79 & -121.94 & 157.51 & -63.95 & 90.52 & 63.60 \\
\hline Beta-7 & 1.42 & 2.29 & 0.68 & 0.43 & -143.90 & 147.29 & 65.86 & -99.83 & -65.53 \\
\hline Beta-7-2 & 0.81 & 1.95 & 1.91 & 0.77 & -145.62 & 149.77 & 66.53 & -95.09 & -179.60 \\
\hline Beta-7-3 & 1.62 & 2.74 & 0.49 & 0.20 & -145.01 & 150.68 & 67.44 & -121.71 & 67.91 \\
\hline Beta-8 & 0.12 & 0.72 & 6.11 & 6.11 & -110.66 & 121.89 & 176.34 & -99.58 & -179.34 \\
\hline Beta-8-3 & 0.67 & 1.15 & 2.43 & 2.97 & -109.71 & 120.63 & 176.52 & -95.99 & -64.38 \\
\hline Beta-9 & 0.27 & 0.78 & 4.78 & 5.57 & -122.63 & 157.20 & -64.13 & -97.17 & -179.47 \\
\hline Beta-9-3 & 0.85 & 1.94 & 1.79 & 0.79 & -121.13 & 156.79 & -64.09 & -92.21 & -63.73 \\
\hline AlphaR-1 & 0.10 & 1.13 & 6.32 & 3.08 & -110.23 & -0.33 & -64.41 & -95.36 & -179.52 \\
\hline AlphaR-1-2 & 0.62 & 1.48 & 2.62 & 1.72 & -109.50 & 1.61 & -64.93 & -115.43 & 65.14 \\
\hline AlphaR-1-3 & 0.58 & 1.69 & 2.83 & 1.19 & -111.56 & 1.77 & -65.28 & -91.58 & -64.10 \\
\hline AlphaR-2 & 0.12 & 1.04 & 6.16 & 3.57 & -110.95 & 3.44 & -65.33 & 100.96 & 179.36 \\
\hline AlphaR-2-2 & 0.62 & 1.58 & 2.64 & 1.44 & -110.10 & 2.37 & -64.92 & 98.47 & 64.57 \\
\hline AlphaR-2-3 & 0.71 & 1.73 & 2.27 & 1.11 & -111.12 & 2.41 & -65.14 & 126.29 & -66.07 \\
\hline AlphaR-3 & 1.89 & 1.94 & 0.31 & 0.78 & -92.90 & -15.19 & 71.24 & 155.68 & 179.26 \\
\hline AlphaR-3-3 & 2.62 & 3.96 & 0.09 & 0.03 & -92.66 & -14.26 & 71.14 & 144.13 & -66.46 \\
\hline AlphaR-4 & 1.91 & 2.48 & 0.30 & 0.31 & -82.38 & -30.53 & 170.59 & 162.89 & 179.57 \\
\hline AlphaR-4-2 & 2.43 & 3.57 & 0.12 & 0.05 & -80.68 & -30.60 & 171.00 & 98.93 & 64.34 \\
\hline AlphaR-4-3 & 2.51 & 3.31 & 0.11 & 0.08 & -81.77 & -30.29 & 171.88 & 126.01 & -65.96 \\
\hline AlphaR-5 & 1.82 & 2.98 & 0.35 & 0.13 & -92.74 & -14.85 & 71.74 & -97.94 & -179.67 \\
\hline AlphaR-5-2 & 2.43 & 3.35 & 0.12 & 0.07 & -93.09 & -14.33 & 71.80 & -113.59 & 65.23 \\
\hline AlphaR-5-3 & 2.38 & 3.31 & 0.14 & 0.08 & -95.34 & -14.35 & 71.48 & -90.79 & -64.60 \\
\hline AlphaR-6 & 1.88 & 2.51 & 0.32 & 0.30 & -95.55 & -11.66 & 72.29 & 100.36 & 179.31 \\
\hline AlphaR-6-2 & 2.46 & 3.79 & 0.12 & 0.03 & -91.94 & -14.04 & 71.24 & 98.84 & 64.39 \\
\hline AlphaR-7 & 1.88 & 2.88 & 0.32 & 0.16 & -84.52 & -30.59 & 170.13 & -93.73 & -179.92 \\
\hline AlphaR-7-2 & 2.70 & 4.39 & 0.08 & 0.01 & -84.61 & -29.20 & 171.55 & -111.54 & 66.14 \\
\hline AlphaR-7-3 & 2.48 & 3.57 & 0.11 & 0.05 & -86.20 & -29.39 & 171.35 & -89.87 & -64.78 \\
\hline AlphaR-8 & 1.96 & 2.90 & 0.28 & 0.16 & -82.30 & -29.15 & 170.51 & 100.92 & 179.41 \\
\hline
\end{tabular}




\begin{tabular}{|c|c|c|c|c|c|c|c|c|c|}
\hline Conf. & $\begin{array}{l}\Delta E_{\mathrm{ZPC}} \\
\left(\mathrm{kcal} \mathrm{mol}^{-1}\right)\end{array}$ & $\begin{array}{l}\Delta \mathbf{G}_{298 \mathrm{~K}} \\
\left(\mathrm{kcal} \mathrm{mol}^{-1}\right)\end{array}$ & $\begin{array}{l}\text { pop- } \Delta E \\
(\%)\end{array}$ & $\begin{array}{l}\text { pop- } \Delta \mathrm{G} \\
(\%)\end{array}$ & $\phi$ & $\psi$ & $\alpha$ & $\rho 1$ & $\rho 2$ \\
\hline AlphaR-8-2 & 2.43 & 3.57 & 0.12 & 0.05 & -80.67 & -30.60 & 171.00 & 98.93 & 64.34 \\
\hline AlphaR-8-3 & 2.51 & 3.31 & 0.11 & 0.08 & -81.77 & -30.29 & 171.88 & 126.01 & -65.96 \\
\hline C7eq-10 & 0.34 & 0.76 & 4.23 & 5.76 & -85.30 & 91.38 & -178.54 & -157.75 & -179.26 \\
\hline C7eq-10-2 & 0.86 & 1.39 & 1.77 & 1.97 & -85.57 & 89.06 & -178.49 & -141.32 & 65.72 \\
\hline C7eq-10-3 & 0.89 & 1.61 & 1.69 & 1.38 & -85.87 & 87.43 & -178.26 & -100.02 & -64.10 \\
\hline C7eq-11 & 2.43 & 3.48 & 0.12 & 0.06 & -85.86 & 76.35 & 56.18 & -99.87 & -179.45 \\
\hline C7eq-11-2 & 3.02 & 4.07 & 0.05 & 0.02 & -85.99 & 78.92 & 55.82 & -127.51 & 65.63 \\
\hline C7eq-11-3 & 2.98 & 4.23 & 0.05 & 0.02 & -85.59 & 77.42 & 56.11 & -97.15 & -63.93 \\
\hline C7eq-12 & 1.52 & 2.86 & 0.58 & 0.17 & -86.17 & 59.44 & -73.11 & -96.12 & -179.44 \\
\hline C7eq-12-2 & 2.09 & 3.21 & 0.22 & 0.09 & -86.36 & 63.99 & -73.93 & -116.79 & 65.41 \\
\hline C7eq-12-3 & 2.01 & 3.34 & 0.25 & 0.07 & -85.98 & 62.14 & -73.67 & -93.07 & -64.17 \\
\hline C7eq-5 & 2.50 & 3.86 & 0.11 & 0.03 & -86.08 & 78.80 & 55.54 & 90.42 & 179.70 \\
\hline C7eq-5-2 & 3.22 & 4.35 & 0.03 & 0.01 & -88.37 & 76.95 & 56.35 & 88.35 & 65.85 \\
\hline C7eq-5-3 & 3.26 & 4.48 & 0.03 & 0.01 & -87.58 & 75.98 & 56.27 & 110.78 & -65.66 \\
\hline C7eq-6 & 1.57 & 2.84 & 0.53 & 0.17 & -86.45 & 63.64 & -73.66 & 93.73 & 179.70 \\
\hline C7eq-6-2 & 2.22 & 3.81 & 0.18 & 0.03 & -87.17 & 59.02 & -72.80 & 89.08 & 64.49 \\
\hline C7eq-6-3 & 2.26 & 3.70 & 0.16 & 0.04 & -87.30 & 60.12 & -72.79 & 115.43 & -65.59 \\
\hline C7ax-1 & 4.76 & 6.36 & 0.00 & 0.00 & 63.62 & -35.61 & -58.87 & -95.56 & -179.48 \\
\hline C7ax-1-2 & 5.32 & 6.64 & 0.00 & 0.00 & 64.05 & -32.91 & -58.06 & -125.94 & 65.84 \\
\hline C7ax-1-3 & 5.22 & 6.70 & 0.00 & 0.00 & 63.91 & -33.95 & -58.07 & -93.54 & -64.20 \\
\hline C7ax-2 & 4.85 & 6.40 & 0.00 & 0.00 & 63.52 & -31.12 & -57.79 & -164.80 & -179.63 \\
\hline C7ax-2-3 & 5.59 & 7.13 & 0.00 & 0.00 & 63.19 & -32.14 & -58.43 & -173.78 & -67.24 \\
\hline C7ax-3 & 5.70 & 7.48 & 0.00 & 0.00 & 63.25 & -37.20 & -61.27 & 113.98 & -67.47 \\
\hline C7ax-3-2 & 5.31 & 6.97 & 0.00 & 0.00 & 62.99 & -33.66 & -58.78 & 91.79 & 65.17 \\
\hline C7ax-3-3 & 4.75 & 6.23 & 0.00 & 0.00 & 63.34 & -33.30 & -58.62 & 91.25 & 179.34 \\
\hline C7ax-4 & 5.68 & 6.89 & 0.00 & 0.00 & 67.62 & -42.33 & 77.14 & -95.42 & -179.48 \\
\hline C7ax-4-2 & 6.26 & 7.50 & 0.00 & 0.00 & 69.08 & -39.86 & 79.68 & -117.08 & 65.69 \\
\hline C7ax-4-3 & 6.23 & 7.55 & 0.00 & 0.00 & 68.42 & -40.69 & 78.68 & -92.02 & -64.40 \\
\hline C7ax-5 & 1.96 & 3.38 & 0.28 & 0.07 & 76.10 & -59.05 & 179.00 & 98.79 & 179.12 \\
\hline C7ax-5-2 & 2.53 & 4.24 & 0.11 & 0.02 & 75.92 & -59.85 & 179.17 & 94.86 & 64.20 \\
\hline C7ax-6 & 2.02 & 3.25 & 0.25 & 0.09 & 75.95 & -62.14 & 178.97 & 166.41 & 179.68 \\
\hline C7ax-6-3 & 2.63 & 3.73 & 0.09 & 0.04 & 76.36 & -61.54 & 179.83 & 135.37 & -66.06 \\
\hline C7ax-7 & 6.76 & 8.35 & 0.00 & 0.00 & 64.86 & -47.16 & 68.26 & 117.82 & -67.77 \\
\hline C7ax-7-2 & 6.37 & 8.06 & 0.00 & 0.00 & 66.30 & -42.35 & 74.35 & 94.91 & 64.92 \\
\hline C7ax-7-3 & 5.71 & 7.06 & 0.00 & 0.00 & 66.82 & -41.82 & 75.80 & 92.17 & 179.31 \\
\hline C7ax-8 & 2.51 & 3.92 & 0.11 & 0.03 & 76.91 & -59.75 & 178.74 & -89.01 & -64.94 \\
\hline C7ax-8-2 & 2.68 & 4.56 & 0.08 & 0.01 & 76.87 & -59.01 & 178.49 & -112.35 & 65.53 \\
\hline C7ax-8-3 & 1.94 & 3.59 & 0.28 & 0.05 & 76.09 & -61.35 & 178.77 & -90.92 & -179.78 \\
\hline C7ax-9 & 5.77 & 7.16 & 0.00 & 0.00 & 68.64 & -38.93 & 80.47 & -167.71 & -179.54 \\
\hline C7ax-9-3 & 6.53 & 7.67 & 0.00 & 0.00 & 67.86 & -39.82 & 78.85 & -175.55 & -66.82 \\
\hline AlphaL-1 & 4.87 & 5.98 & & & & & & & \\
\hline Cno-1 & 6.54 & 7.39 & & & & & & & \\
\hline
\end{tabular}



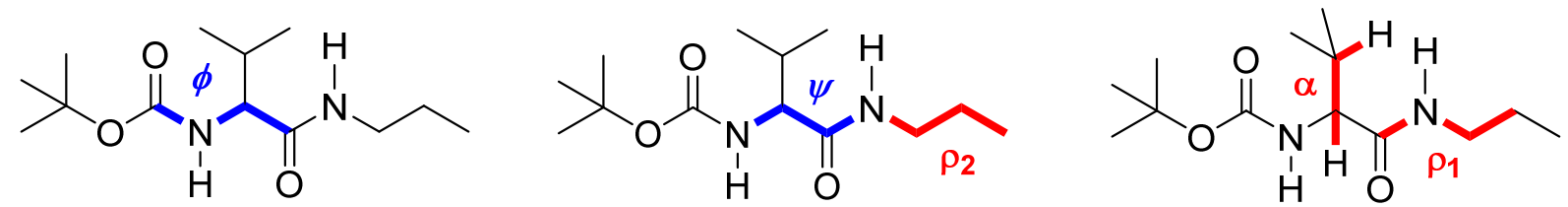

Scheme S1. Angle definitions used in the conformational analysis and in Table S1.

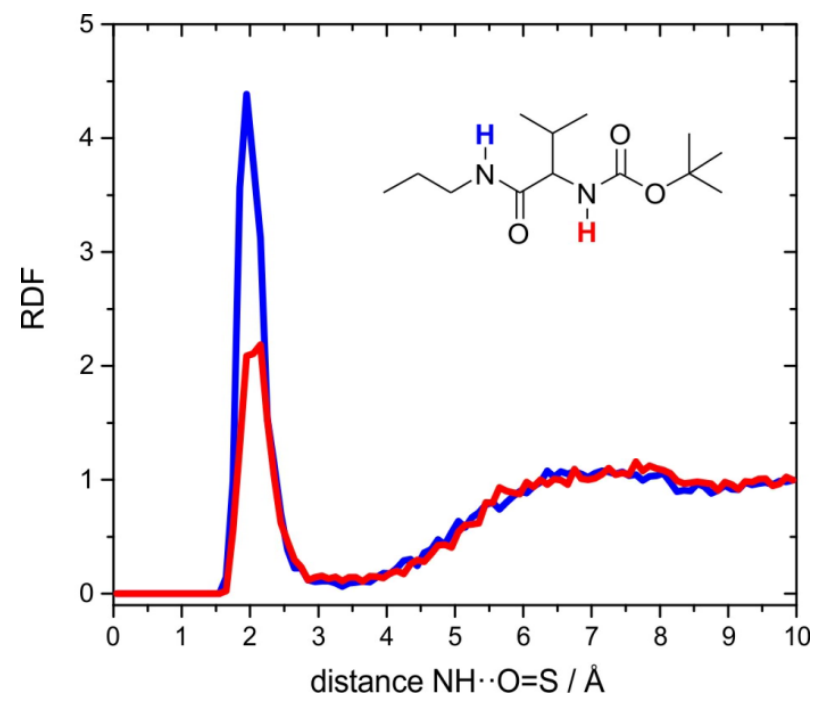

Figure S1. Radial distribution function for the interaction of the NH-groups with single DMSO molecules through an $\mathrm{NH} \cdot \cdot \mathrm{O}=\mathrm{S}\left(\mathrm{CH}_{3}\right)_{2}$ hydrogen bond. 


\section{Additional data for 1 in chloroform solution}
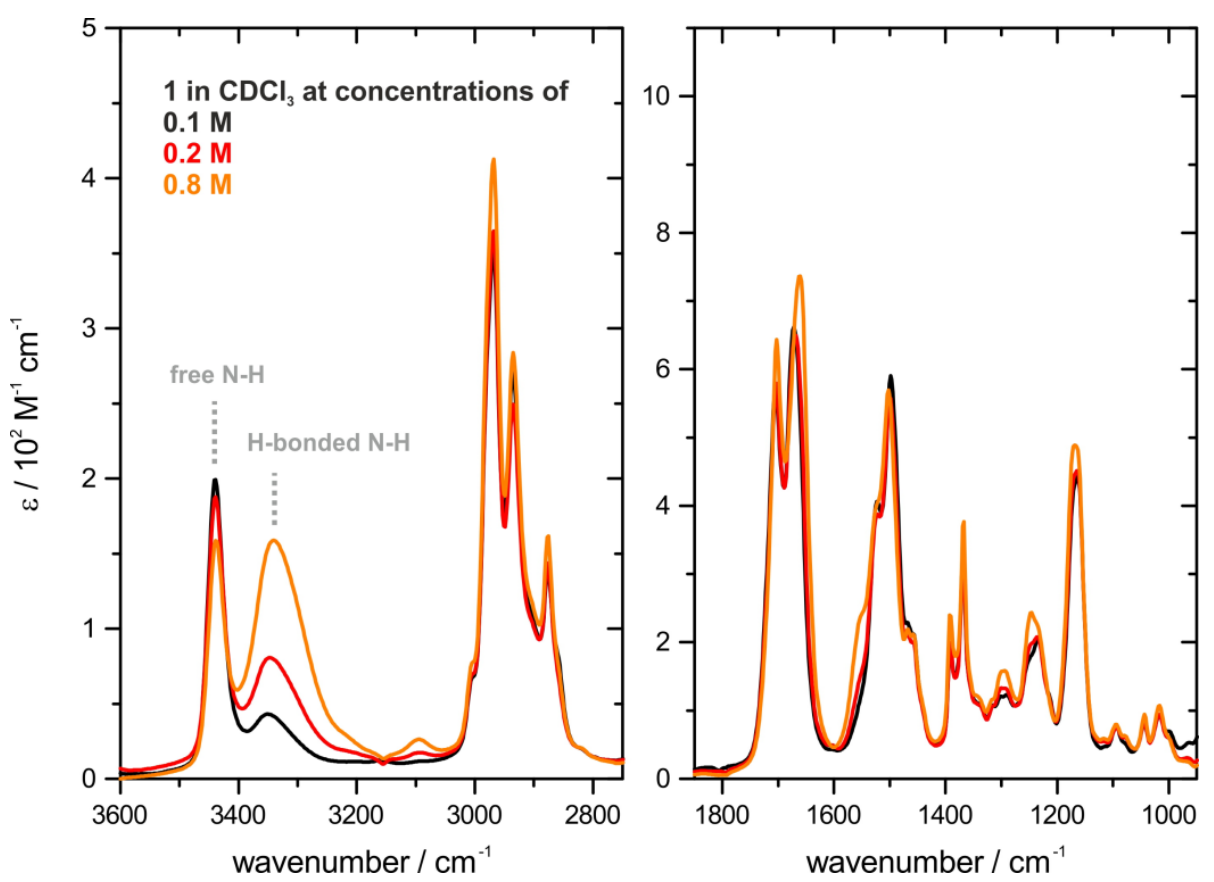

Figure S2. Concentration dependence of the IR spectra of $\mathbf{1}$ in $\mathrm{CDCl}_{3}$.

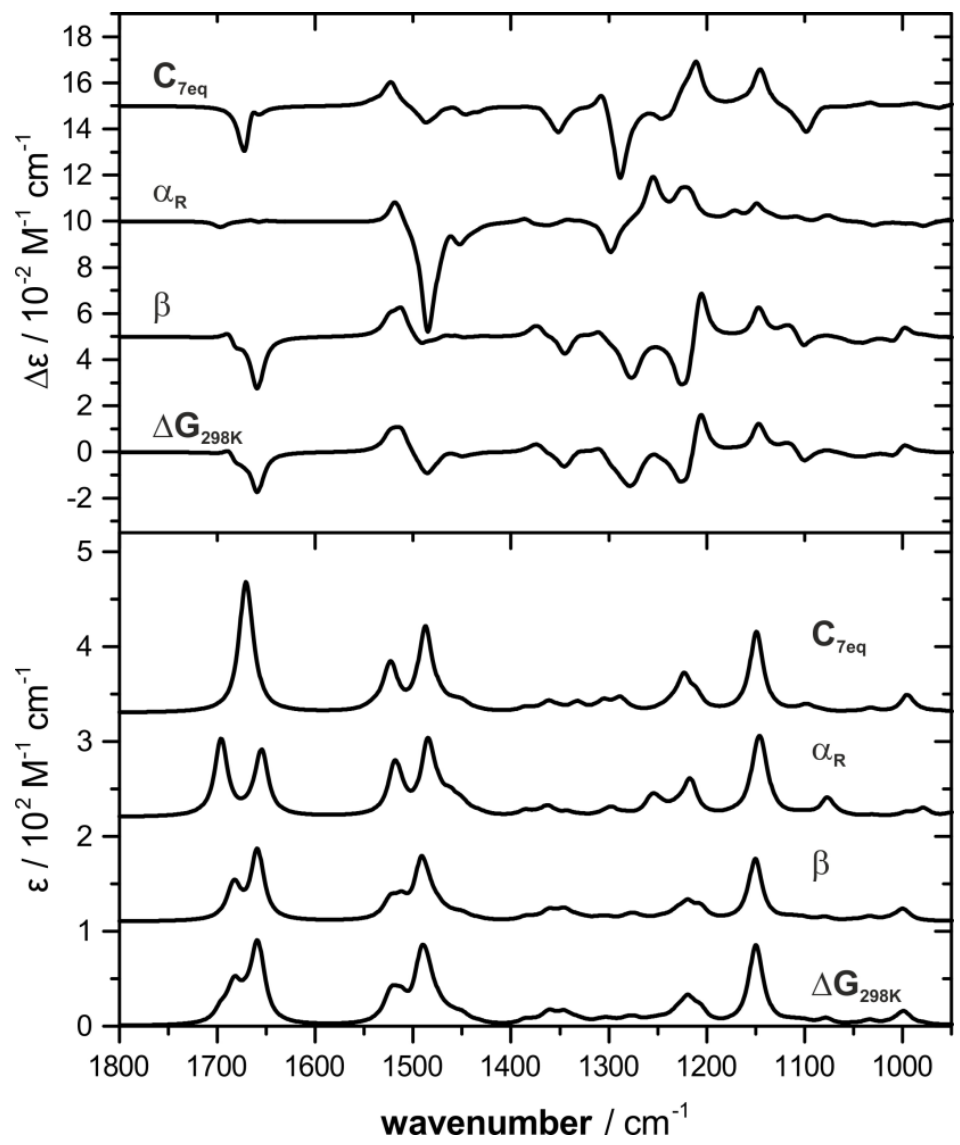

Figure S3. IR and VCD spectra of the individual conformer classes 


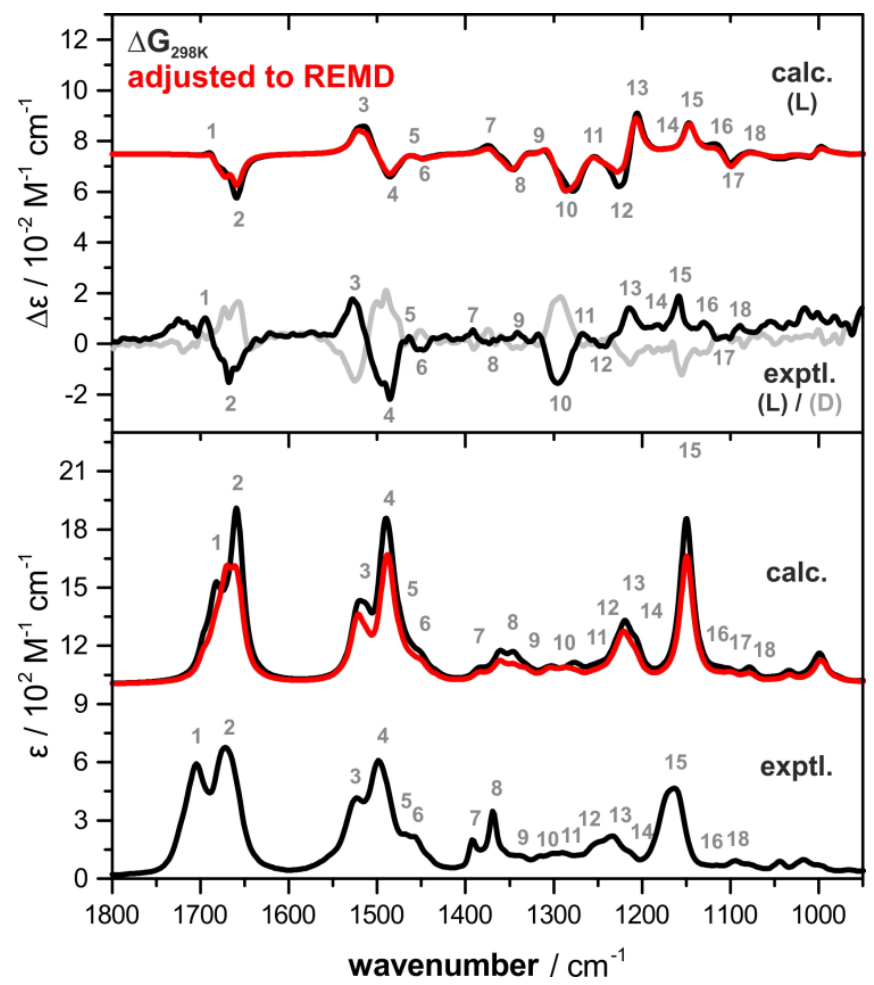

Figure S4. Comparison of experimental IR and VCD spectra to the calculated spectra based on the REMD conformational distribution. 


\section{Additional data for 1 in DMSO}

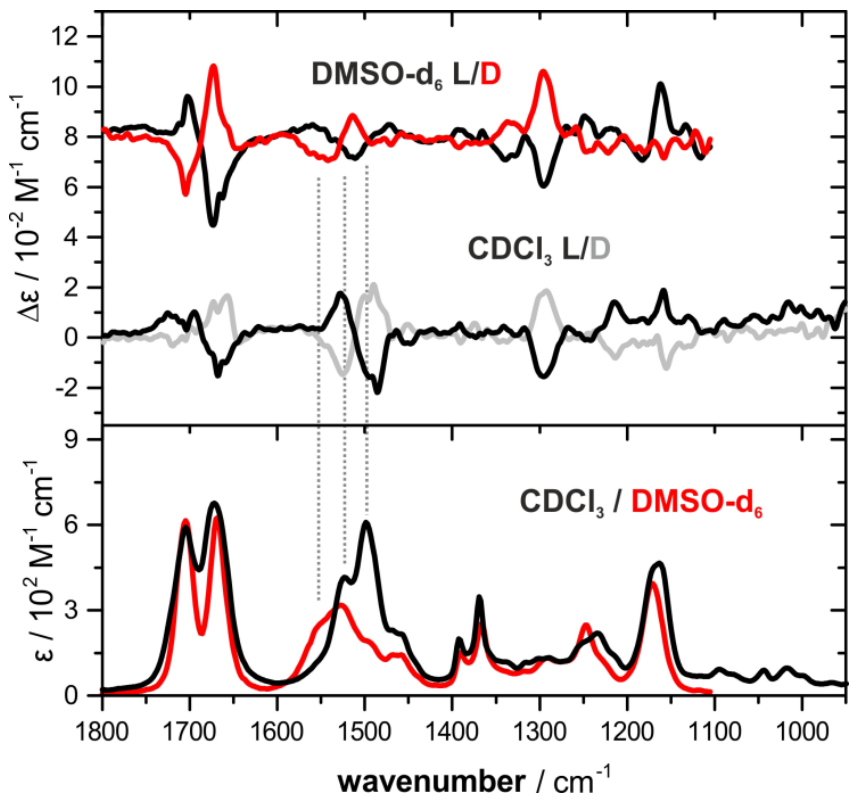

Figure S5. Direct comparison of experimental spectra of 1 in $\mathrm{CDCl}_{3}$ and DMSO- $\mathrm{d}_{6}$.
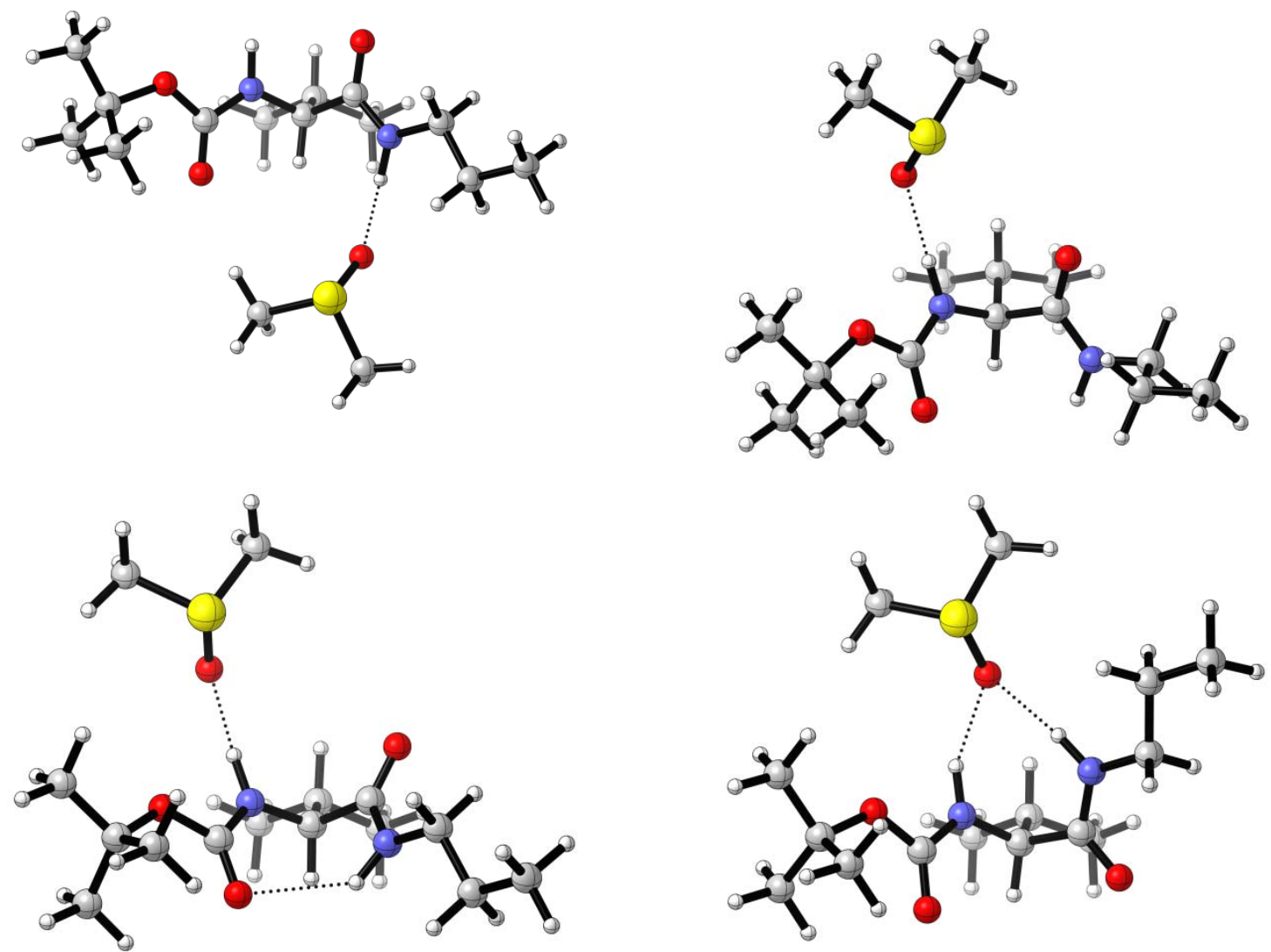

Figure S6. DMSO-solvated structures of the $\beta-, \mathrm{C}_{7 \mathrm{eq}^{-}}$, and $\alpha_{\mathrm{R}}$-conformer 


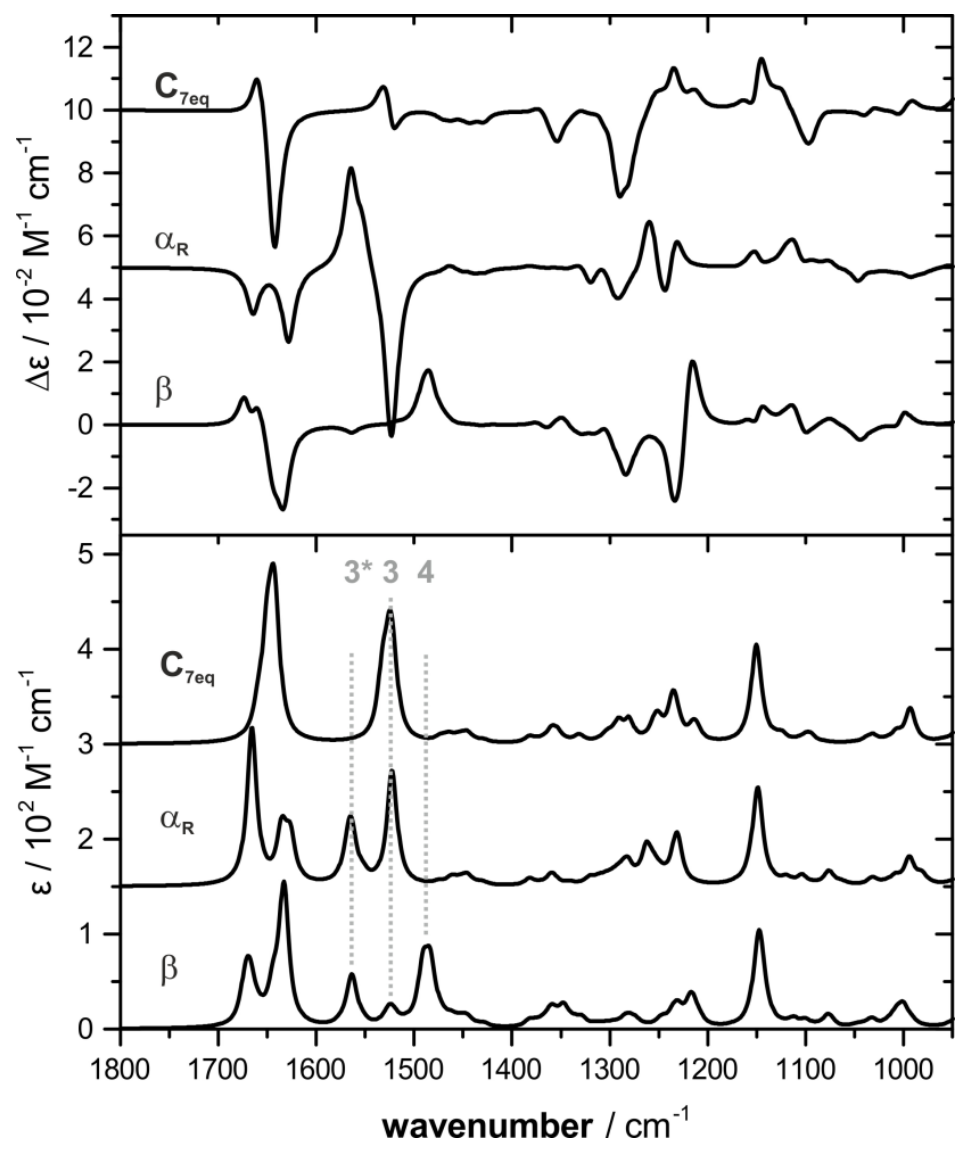

Figure S7. IR and VCD spectra of the individual conformer classes for explicitly solvated [1·DMSO] 


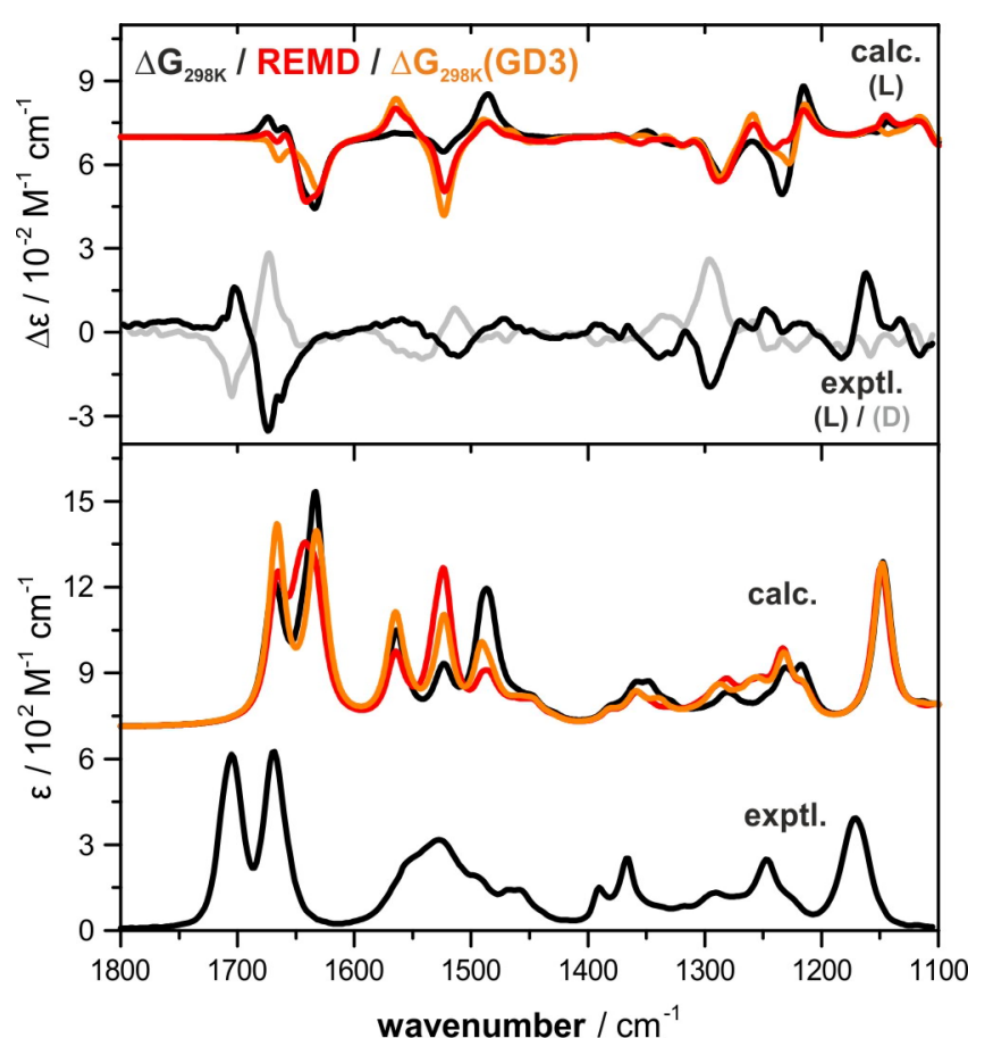

Figure S8. Comparison of the calculated spectra of mono-solvated L-1 with the experimental spectra. 


\section{Additional data on the chiral solvation of 1}

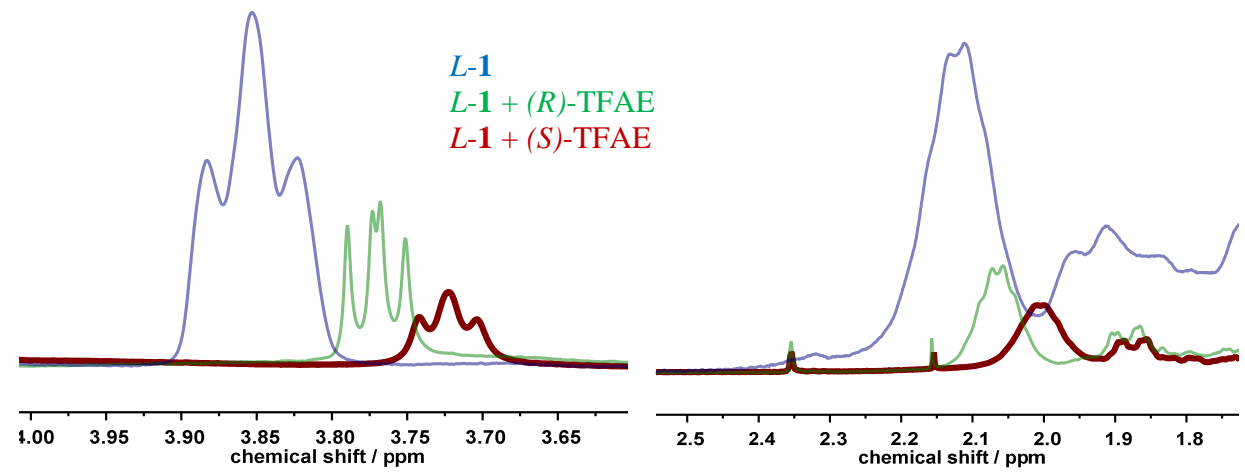

Figure S9. NMR spectra of $L-1$ showing the shift of the $C^{\mathrm{a}}$-H (left) and isopropyl-CH peaks (right) upon addition of one equivalent of either (S)- or (R)-TFAE

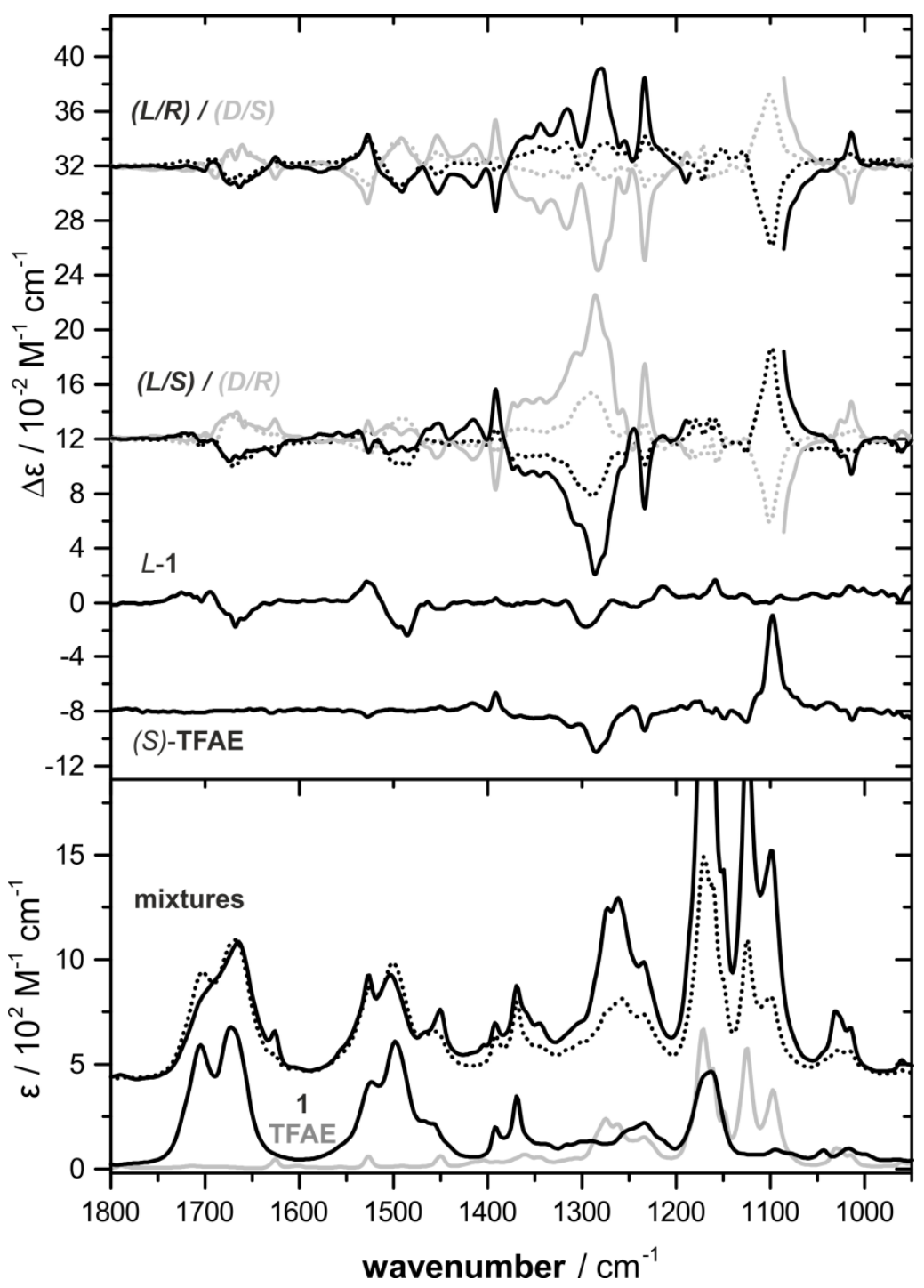

Figure S10. Comparison of the IR and VCD spectra of $L-1$ with those of the 1:1 (dotted) and 1:4 mixtures (solid lines) of $\mathbf{1}$ and TFAE. 


\section{The IR and VCD spectra of TFAE}

Due to its relatively simple structure, TFAE does not feature many strong bands in the fingerprint region of the IR spectrum (cf. Figure S11). Intense bands can be observed beginning from $\sim 1400 \mathrm{~cm}^{-1}$ and below, with the most prominent bands arising from $\mathrm{CH}$ bending vibrations (1300-1200 $\mathrm{cm}^{-1}$ ), $\mathrm{CH}$ in-plane deformations within the antracenyl group (1200-1150 $\left.\mathrm{cm}^{-1}\right)$, and C-O and C-F stretching vibrations (1150-1070 $\mathrm{cm}^{-1}$ ). The VCD spectra of the two enantiomers of TFAE show nice mirror image behavior, and high VCD intensity for those bands which are also strong in the IR. The bands at 1625, 1526, and $1450 \mathrm{~cm}^{-1}$ are also noteworthy, as they feature quite some VCD intensity in comparison to their weak IR bands.

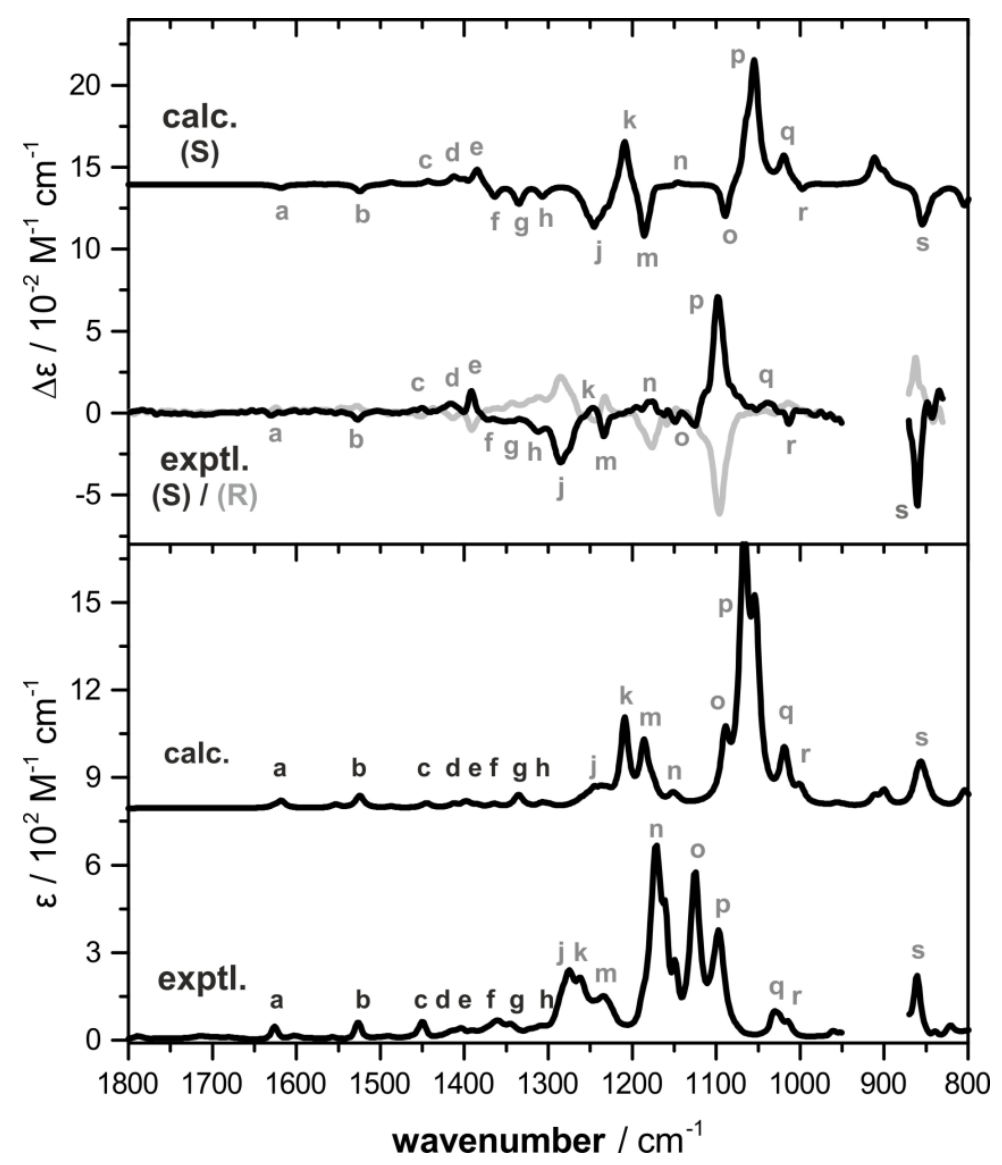

Figure S11. Comparison of the experimental and calculated IR and VCD spectra of TFAE. The letters indicate band assignments.

The conformational preferences of TFAE can also be easily characterized as it can only adopt two different conformations which differ in the relative orientation of the OH-group. Here, conformer 1 $\left(\angle\left(\mathrm{C}_{\mathrm{Ar}}-\mathrm{C}^{\alpha}-\mathrm{O}-\mathrm{H}\right)=160^{\circ}\right)$ is about $0.2 \mathrm{kcal} / \mathrm{mol}$ more stable than conformer $2\left(\angle\left(\mathrm{C}_{\mathrm{Ar}}-\mathrm{C}^{\alpha}-\mathrm{O}-\mathrm{H}\right)=57^{\circ}\right)$. Figure S11 shows the IR and VCD spectra obtained by Boltzmann weighted averaging of the single conformer spectra $\left(\Delta \mathrm{G}_{298 \mathrm{~K}}\right)$. Although many IR bands are predicted to be much weaker than observed in the experiment, the relative intensities of the VCD bands are resembled quite well. 
It is important to note that, in contrast to the simulated spectra of $\mathbf{1}$, the linear frequency scaling factor of 0.975 employed throughout this study shifts most bands of TFAE to significantly too low wavenumbers. While, for instance, mode $e$ is only about $5 \mathrm{~cm}^{-1}$ off compared to the experimental position, modes $j$ and $p$ are already 40 respectively $44 \mathrm{~cm}^{-1}$ too low. A better agreement could be achieved if the frequencies were not scaled at all. Using a factor of 1.01, good agreement in the range of modes $\mathrm{j}$-m could be obtained, while modes $\mathrm{o}$ and $\mathrm{p}$ would require a scaling by 1.05 . While this would generally not cause major concern, it will become important for the calculations of the mixtures. Here, a uniform scaling factor has to be chosen as both $\mathbf{1}$ and TFAE essentially become one entity. Now consider, for instance, VCD bands which cancel in the experimental spectra due to opposite sign. If they are not predicted at roughly the same frequency as well, the calculated bands might give rise to completely artificial VCD spectral pattern in the calculations which do not agree at all with the experimental data. We therefore decided not to attempt the simulation of the vibrational spectra of the mixtures. 


\section{Conformational analysis of [1-TFAE]}

For the conformational analsyis of [1-TFAE], we assumed the strongest interaction between them to occur between the $\mathrm{N}-\mathrm{H}$ and $\mathrm{C}=\mathrm{O}$ of the amide functionalities and the $\mathrm{OH}$-group of the chiral alcohol. Furthermore, we based the conformational analysis solely on the $\beta$-conformation of $\mathbf{1}$ as it facilitates the presumably most ideal hydrogen bonding donor and acceptor orientations, for which two binding topologies featuring either a seven-membered or a nine-membered ring structure can be proposed (Scheme S2).

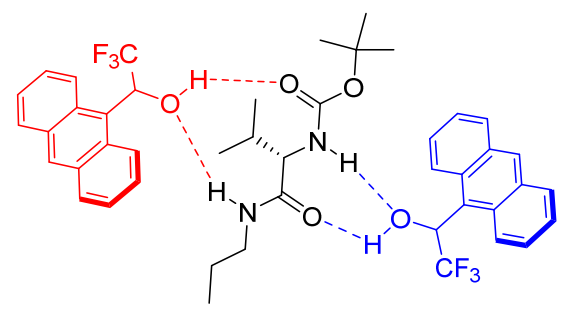

Scheme S2. Proposed interactions between selector and selectand through a seven-membered ring (blue) or a nine-membered ring (red).

Assuming that the influence of the conformationally flexible n-propyl chain on the VCD spectrum is negligible, we considered only conformers with a stretched trans-conformation $\left(\rho_{2}=180^{\circ}\right)$. Despite this simplification, there are still 72 theoretically possible conformers of each interacting pair, $L-\mathbf{1} /(S)$ TFAE and $L-\mathbf{1} /(R)$-TFAE: 9 conformers of the 1, two binding topologies, two conformers of TFAE, and two lone pairs of the TFAE OH-group to interact with.

Interestingly, during the geometry optimization process of the $L-\mathbf{1} /(S)$-TFAE complexes, it was found that a change in the conformation of TFAE always resulted in a change of the interacting oxygen lone pair. Therefore, in total, only 30 conformers of this complex were obtained. The lowest energy conformations of each of the four binding topologies are shown in Figure S12. Besides the conformation of the n-propyl side chain, the key difference between the two structures is the accepting lone pair in the $\mathrm{NH} \cdot \cdot \mathrm{O}$ hydrogen bond. In the case of the $\beta(7, \mathrm{pR})$ binding topology, the antracenyl ring is oriented away from the bulky iso-propyl group of $\mathbf{1}$, while the $\beta(7, \mathrm{pS})$ orientation brings it in closer proximity to it. Similar orientations are also possible in the nine-membered ring topology. 


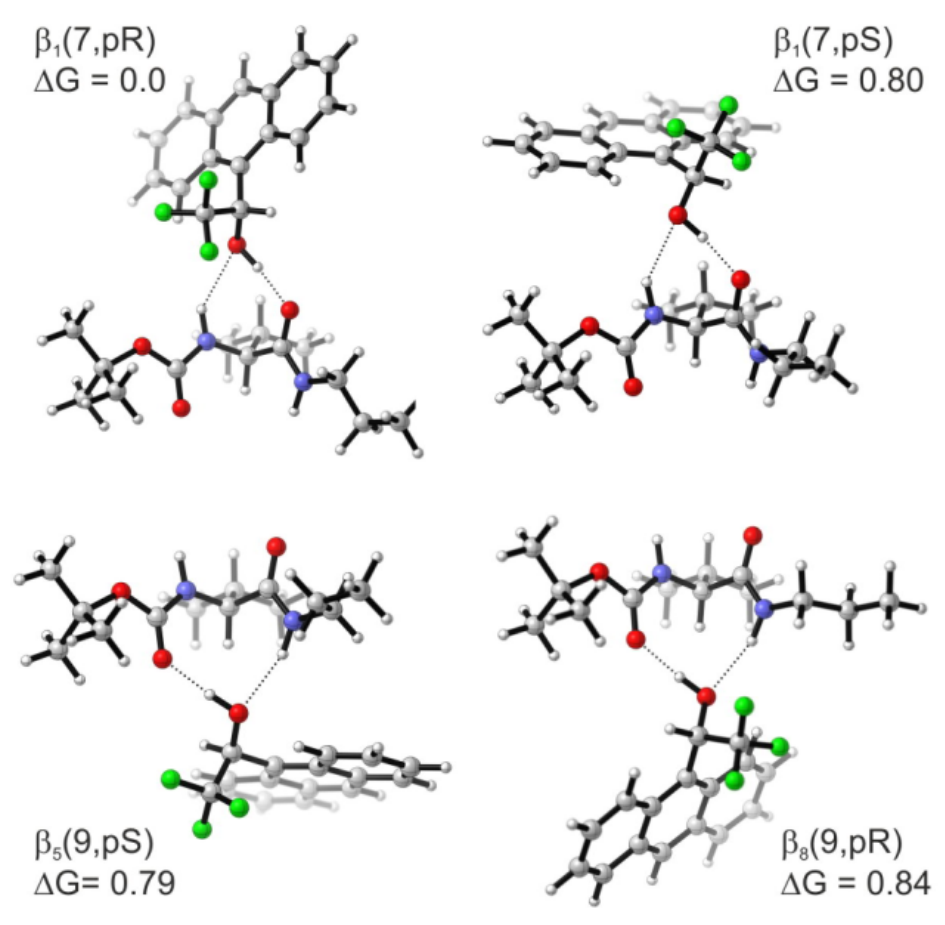

Figure S12. Representative lowest energy structures of complexes of $L-1$ and (S)-TFAE in the sevenand nine-membered ring topologies.

The $L-\mathbf{1} /(S)$-TFAE complexes were used as starting structures for the generation of input structures for the diastereomeric complexes. During preparation, it became obvious that the alcohol cannot adopt its second conformation in all binding topologies due to steric repulsion of the bulky $\mathrm{CF}_{3^{-}}$and antracenyl-group. For instance, inverting the stereocenter of TFAE in the $\beta_{1}(7 \mathrm{pS})$ structure shown in Figure S12, and adjusting the $\mathrm{C}_{\mathrm{Ar}}-\mathrm{C}^{\alpha}-\mathrm{O}-\mathrm{H}$ angle to a preferred conformation of (R)-TFAE (i.e. from $\sim+140^{\circ}$ in (S)-TFEA to $-140^{\circ}$ for ( $R$ )-TFAE), the $\mathrm{CF}_{3}$ group will feel significant steric repulsion from the iso-propyl group. The easiest way for the system to relax this unfavorable interaction appears to be changing the binding lone pair of the oxygen. Thus, for the $L-\mathbf{1} /(R)$-TFAE complex, only $\beta(7 \mathrm{pR})$ and $\beta(9 \mathrm{pS})$ conformers were obtained, totaling to only 15 structures. The cumulative contributions of each binding topology are summarized in Table S2.

\begin{tabular}{|c|c|c|c|c|c|c|}
\hline \multicolumn{7}{|c|}{$\begin{array}{l}\text { Table S2. Contributions of each binding topology to the overall populations } \\
\text { of the mixture populations of the } L-1 \text { based on } \Delta \mathrm{E}_{\mathrm{ZPC}} \text { and } \Delta \mathrm{G}_{298 \mathrm{~K}} \text {. }\end{array}$} \\
\hline & \multicolumn{3}{|c|}{$L-1 /(S)$-TFAE } & \multicolumn{3}{|c|}{$L-1 /(R)$-TFAE } \\
\hline & $\Sigma(\Delta \mathrm{E})$ & $\Sigma(\Delta \mathrm{G})$ & $\begin{array}{l}\text { no. } \\
\text { conf. }\end{array}$ & $\Sigma(\Delta \mathrm{E})$ & $\Sigma(\Delta \mathrm{G})$ & no. conf. \\
\hline$\beta(7 \mathrm{pR})$ & 21.76 & 49.4 & 9 & 61.32 & 67.65 & 9 \\
\hline$\beta(7 \mathrm{pS})$ & 24.88 & 28.57 & 9 & & & \\
\hline$\beta(9 \mathrm{pS})$ & 37.75 & 9.72 & 6 & 38.68 & 32.35 & 6 \\
\hline$\beta(9 \mathrm{pR})$ & 15.62 & 12.31 & 6 & & & \\
\hline
\end{tabular}

